\title{
Introduction to the Special Issue of JCP-A featuring the Presidential Symposium at the International Congress of Neuroethology, ICN 2016 in Montevideo
}

\author{
P. M. Narins ${ }^{1}$
}

Published online: 13 July 2017

(C) Springer-Verlag Berlin Heidelberg 2017

The idea behind the Inaugural Presidential Symposium during the 12th International Congress of Neuroethology that took place in March, 2016 in Montevideo, Uruguay, was to showcase the wide range of outstanding current studies being carried out around the world on the neural bases of behavior. My primary goal was to invite speakers doing outstanding, creative, cutting-edge work. Equally important was that I had to have been bowled over by both their Science and their presentations! This would ensure a broad appeal to both the non-specialists and the specialists who wished to learn and affirm what constitutes modern "neuroethology."

The Presidential Symposium plenary session was scheduled as the opening salvo of the Congress. Moreover, each of the presenters was invited to produce a manuscript at least loosely based on their talk and to submit it for peer review to be published in a Special Issue of the Journal of Comparative Physiology-A. This journal has a long history of close to 100 years of publishing original papers in the field of comparative physiology and behavior, and has been a strong supporter of the field of neuroethology since its inception. In fact, a substantial portion of the travel costs for the Presidential Symposium speakers attending the Congress was offset by Springer-Verlag as well as by the International Society for Neuroethology and a generous personal contribution from Professor Friedrich and Ortrun Barth. We hope that these contributors (and others) will continue their critical support for the Presidential

P. M. Narins

pnarins@ucla.edu

Departments of Integrative Biology \& Physiology, and Ecology \& Evolutionary Biology, University of California Los Angeles, Los Angeles, CA 90095, USA
Symposium in future congresses of the International Society of Neuroethology.

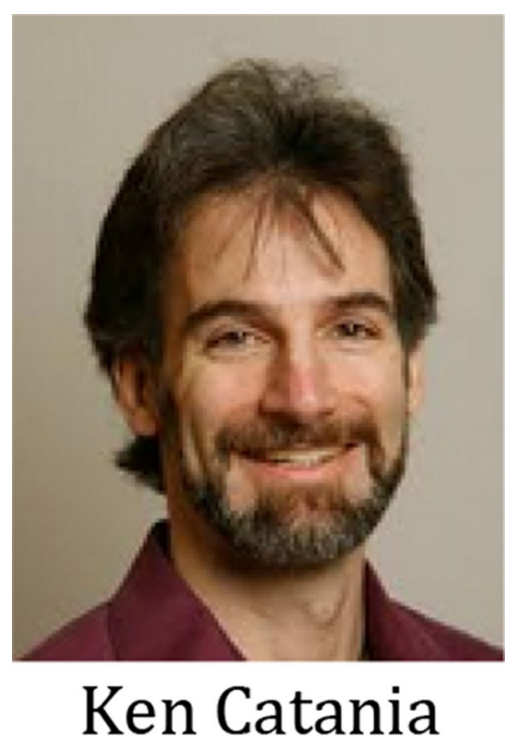

The first speaker in the Presidential Symposium was Kenneth C. Catania of Vanderbilt University in Nashville, Tennessee, USA. His paper in this Special Issue is entitled: "Behavioral pieces of neuroethological puzzles." In his review, Catania provides a fascinating first-person account of surprising insights that have come from the behavioral dimension of neuroethological studies in his laboratory. These studies include the early attempts to understand the function of the nose in star-nosed moles and to explore its representation in the neocortex. This led to the discovery of a somatosensory fovea that parallels the visual fovea of primates in several ways. Subsequent experiments to investigate the assumed superiority of star-nosed moles to their relatives when locating food led to the unexpected 
discovery of stereo olfaction in common moles. The exceptional olfactory abilities of common moles, in turn, helped to explain an unusual bait-collecting technique called "worm-grunting" in the American southeast. Finally, the predatory behavior of tentacled snakes was best understood not by exploring their nervous system, but rather by considering fish nervous systems. These experiences highlight the difficulty of predicting the abilities of animals that have senses foreign to the investigator, and also the rewards of discovering the unexpected.

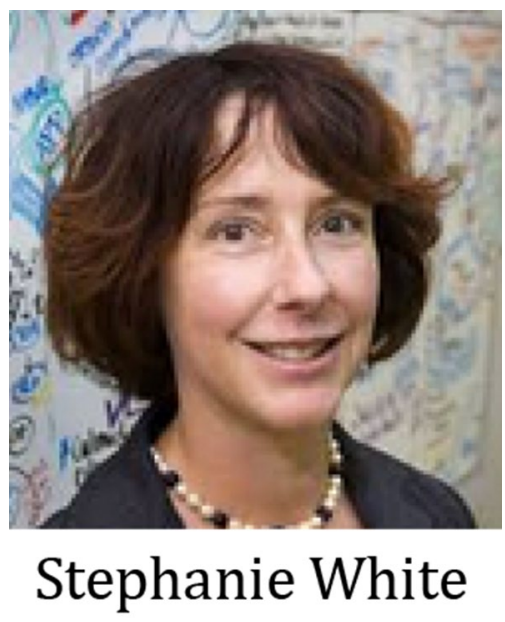

The next speaker was Stephanie A. White from the University of California Los Angeles, Los Angeles, California, USA. Her paper, with Jonathan B. Heston as co-author, deals with one of the classic neuroethological modelsvocal learning in the songbird. Their contribution is entitled: "To transduce a zebra finch: interrogating behavioral mechanisms in a model system for speech." The ability to alter neuronal gene expression, either to affect levels of endogenous molecules or to express exogenous ones, is a powerful tool for linking brain and behavior. Thousands of bird species exhibit the ability to imitate sounds, a trait known as vocal learning which also is a feature of human speech. The circuits and underlying molecular mechanisms appear similar between disparate avian orders and are shared with humans. One advantage of studying vocallearning birds is that the neurons dedicated to this trait are nested within the surrounding brain regions, providing discrete anatomical targets for relating brain and behavior. In songbirds, these nuclei are known collectively as the song control system. The authors have exploited the ability of viruses to insert genetic material into neurons to drive the expression of experimenter-defined genes, and in this way interrogate molecular function in non-traditional model organisms. To date, the use of viruses in the song control system is in its infancy. In this paper, the authors review prior successes and test additional viruses for their capacity to transduce basal ganglia song control neurons. In the authors' words, "These findings provide a roadmap for troubleshooting the use of viruses in animal champions of fascinating behaviors"

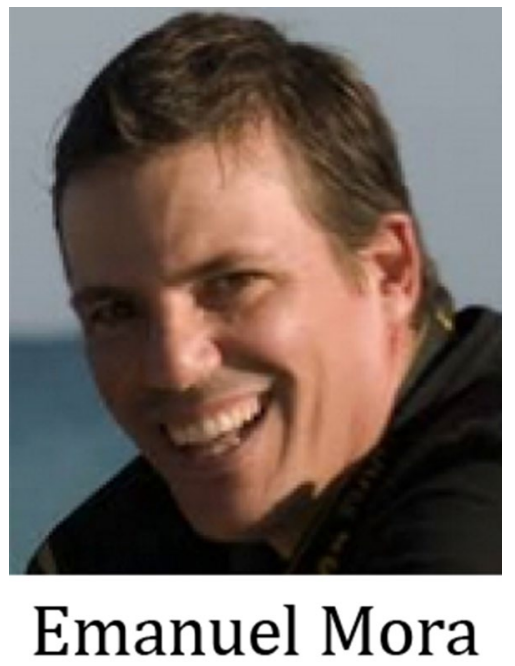

Our next speaker was Emanuel Mora from the University of Havana, Cuba. In their paper entitled: "Hearing diversity in moths confronting a neotropical bat assemblage," Ariadna Cobo-Cuan, Manfred Kössl, and Emanuel Mora present a timely example of the close links between the acoustic sensory abilities of a vertebrate predator (bat) and its invertebrate prey (moth). The tympanal ear is an evolutionary acquisition which aids moths in their survival from bat predation. The greater diversity of bats and echolocation strategies in the Neotropics compared with temperate zones might be expected to impose different sensory constraints on the neotropical moths. However, even given some variability among moth assemblages, the frequency ranges of best hearing of moths from different climate zones studied to date have been roughly the same: between 20 and $60 \mathrm{kHz}$. The authors have analyzed the auditory characteristics of tympanate moths from Cuba, a neotropical island with high levels of bat diversity and a high incidence of echolocation frequencies above those commonly at the upper limit of moths' hearing sensitivity. Moths of the superfamilies Noctuoidea, Geometroidea and Pyraloidea were examined. Audiograms were determined by non-invasively measuring distortion-product otoacoustic emissions. The frequency spectra of the bat echolocation sounds to which this moth community is exposed were also quantified. The hearing ranges of moths in this study showed best frequencies between 36 and $94 \mathrm{kHz}$. High sensitivity to frequencies above $50 \mathrm{kHz}$ suggests that the auditory sensitivity of moths "tracks" the sounds used by the sympatric echolocating bat fauna. The message here is that despite the high biodiversity that characterizes predators and prey in the Neotropics, bat-moth acoustic interactions are dynamically maintained and spectrally matched. 


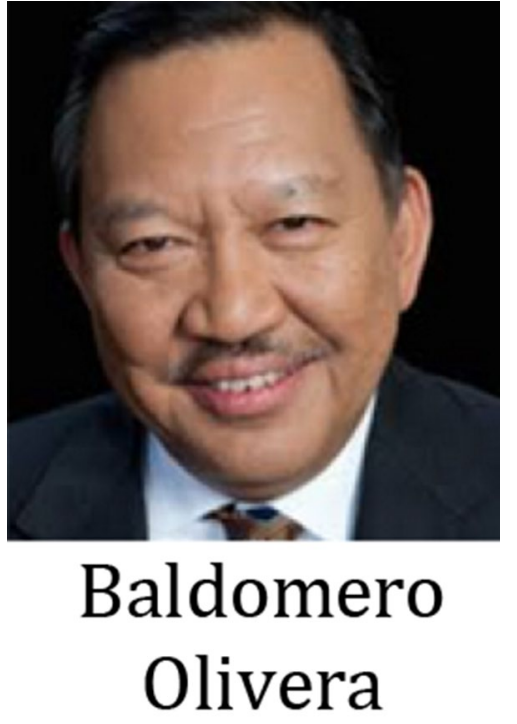

The fourth speaker in the Presidential Symposium was Baldomero Olivera from the University of Utah, in Salt Lake City, Utah, USA. His paper is entitled: "Linking neuroethology to the chemical biology of natural products interactions between cone snails and their fish prey, a case study" and co-authored by Shrinivasan Raghuraman, Eric W. Schmidt, and Helena Safavi-Hemami. The authors investigate the neuroethological underpinnings of an invertebrate predator (cone snail) and its vertebrate prey (fish). And their rationale for doing so is quintessentially neuroethological: "Natural products hold tremendous potential as drug leads and have been extensively studied by chemists and biochemists in the pharmaceutical industry. However, the biological purpose for which a natural product evolved is rarely addressed. By focusing on a well-studied group of natural products-venom components from predatory marine cone snails-this review provides a rationale for why a better understanding of the evolution, biology and biochemistry of natural products will facilitate both neuroscience and the potential for drug leads. The larger goal is to establish a new sub-discipline in the broader field of neuroethology that we refer to as 'Chemical Neuroethology', linking the substantial work carried out by chemists on natural products with accelerating advances in neuroethology."

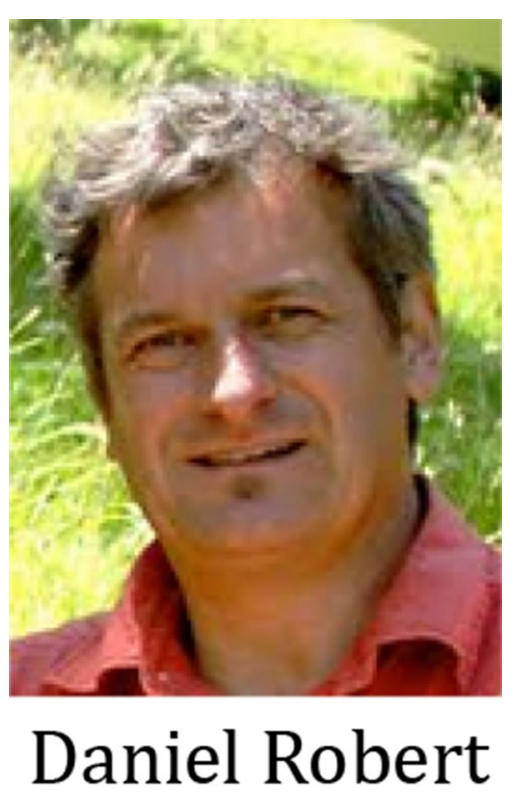

The next speaker was Daniel Robert from the School of Biological Sciences, University of Bristol, UK. In this Special Issue, he and his colleagues present their work entitled: "The bee, the flower and the electric field: electric ecology and aerial electroreception," by Dominic Clarke, Erica Morley, and Daniel Robert. From their abstract we learn: "Bees and flowering plants have a long-standing and remarkable co-evolutionary history. Flowers and bees evolved traits that enable pollination, a process that is as important to plants as it is for pollinating insects. From the sensory ecological viewpoint, bee-flower interactions rely on senses such as vision, olfaction, humidity sensing and touch. Recently, another sensory modality has been unveiled, the detection of the weak electrostatic field that arises between a flower and a bee." The authors present their latest understanding of how these electric interactions arise and how they contribute to pollination and electroreception. Finite element modeling and experimental evidence offer new insights into how these interactions are organized, and how they can be further studied. Focusing on pollen transfer, the authors deconstruct some of the salient features of the three ingredients that enable electrostatic interactions, namely the atmospheric electric field, the capacity of bees to accumulate positive charge, and the propensity of plants to be relatively negatively charged. 


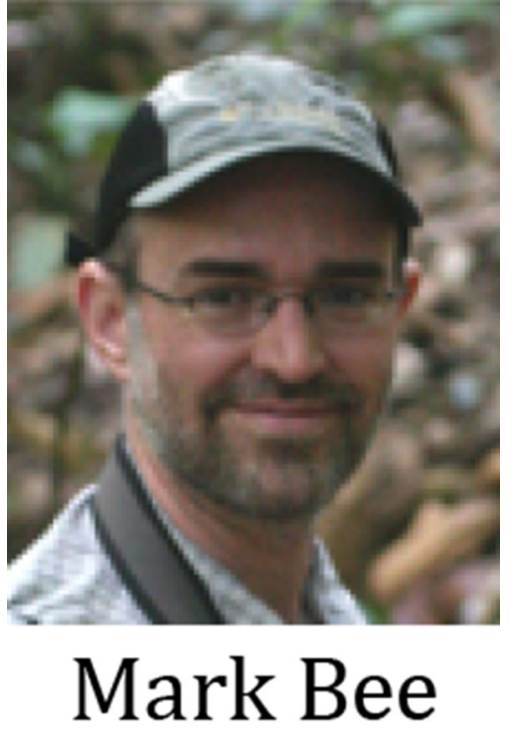

The last speaker in the Presidential Symposium was Tetsuro Matsuzawa from the Section of Language and Intelligence 1, Kyoto University, Kyoto, Japan. His talk was entitled: "The evolutionary origins of human cognitive development: Insights from research on chimpanzees." He spoke brilliantly of his fascinating studies that include tool use in wild chimpanzees in Guinea, West Africa, and numerosity in chimpanzees in the laboratory. Dr. Matsuzawa was unable to contribute a paper to this volume, and so the last paper in this Special Issue is authored by Mark Bee and colleagues from the University of Minnesota, St. Paul, Minnesota, USA. Mark had been invited to participate in the Presidential Symposium, but for personal reasons was unable to attend the Congress in South America. The Special Issue contribution from Mark and his colleagues is entitled: "Nonlinear processing of a multicomponent communication signal by combination-sensitive neurons in the inferior colliculus," by Norman Lee, Katrina M. Schrode and Mark A. Bee. These authors present the results of a neuroethological investigation of sound communication in amphibians and the neural processing of multicomponent communication signals in the anuran midbrain. Their objectives were to evaluate behavioral responses (phonotaxis) to synthetic calls with bimodal and unimodal spectra by females of the green tree frog ( Hyla cinerea) and to describe the responses of single neurons in their midbrains to the same sounds. They found that in phonotaxis trials, only bimodal calls reliably elicited responses, and that these were preferred over unimodal alternatives. Single neurons in the inferior colliculus were classified as combination-insensitive units or combination-sensitive units based on patterns of relative responses to the same bimodal and unimodal calls. Most combination-sensitive units exhibited nonlinear responses (expansive summation, compressive summation, or suppression) to the spectral combination represented in the bimodal call. This indicates that these units likely function in the integrative processing of multiple signal components. Together, their results suggest nonlinearities likely play critical roles in the neural processing of multicomponent communication signals.

It is our hope that this Presidential Symposium and the resulting papers featured in this issue of JCP-A will inspire young scientists and seasoned researchers alike, to carry out neuroethological studies on a wide variety of organisms. August Krogh (1874-1949) wrote that: "For a large number of problems, there will be some animal of choice... on which it can be most conveniently studied," or "Organisms that exhibit extremes of adaptation may reveal general principles not readily observable in less extreme species." I believe that these thoughts beautifully capture the spirit of neuroethology that is amply demonstrated by each presentation at the Presidential Symposium and in each of the contributions to the present Special Issue.

Peter M. Narins, Guest Editor

Immediate Past President of the International Society for Neuroethology. 